\title{
NOUVELLE
}

\section{Les porines : un rôle vital et un lien social au sein du biofilm de Providencia stuartii}

Mariam El-Khatib, Guillaume Tetreau, Jacques-Philippe Colletier
Université Grenoble-Alpes - CEA - CNRS UMR5075, Groupe Dynamique et cinétique des processus moléculaires (DYNAMOP), 71, rue des martyrs - CS 10090 , 38044 Grenoble Cedex 9, France. jacques-philippe.colletier@univ-grenoble-alpes.fr colletier@ibs.fr
> Le terme «biofilm » désigne des communautés multicellulaires enchâssées dans une matrice commune qui les attache à une surface. Dans l'écrin de cette matrice auto-sécrétée, composée de polymères saccharidiques, nucléiques et protéiques, les bactéries du biofilm présentent une résistance accrue aux agressions extérieures et survivent dans des conditions que leurs homologues planctoniques (hors biofilm) ne supporteraient pas [1]. Cela permet aux biofilms d'être omniprésents dans l'environnement, notamment dans des lieux où la contamination peut avoir des consé-

quences délétères sur les activités et la santé humaines, tels que les conduits d'aération, les systèmes de refroidissement des usines ou, dans le milieu hospitalier, les cathéters, prothèses, pacemakers et autres matériels

$(\rightarrow)$ Voir la Synthèse de D. Lebeaux et J.M. Ghigo, $m / s n^{\circ} 8-9$ août-septembre 2012, page 727 médicaux [2] $(\rightarrow)$.

Les biofilms sont ainsi à l'origine de nombreuses infections nosocomiales, parfois devenues chroniques du fait de leur forte résistance au système immunitaire et à l'antibiothérapie. En France, les infections nosocomiales (IN) touchent un patient hospitalisé sur 20, causant plus de 4000 décès chaque année ${ }^{l}$. Un défi pour la microbiologie clinique moderne est donc de développer de nouvelles stratégies visant à cibler et éradiquer les biofilms. Parmi les espèces impliquées dans la formation de telles structures, on retrouve la bactérie Providencia stuartii, un pathogène opportuniste à Gram négatif relativement peu étudié, quoiqu'il représente $10 \%$ des

\footnotetext{
1 Enquête nationale de prévalence 2017 des infections nosocomiales et des traitements anti-infectieux en établissements de santé. Guide de l'enquêteur. Saint Maurice : Santé publique France, $2017: 87$ p. www.santepublique.fr
} 


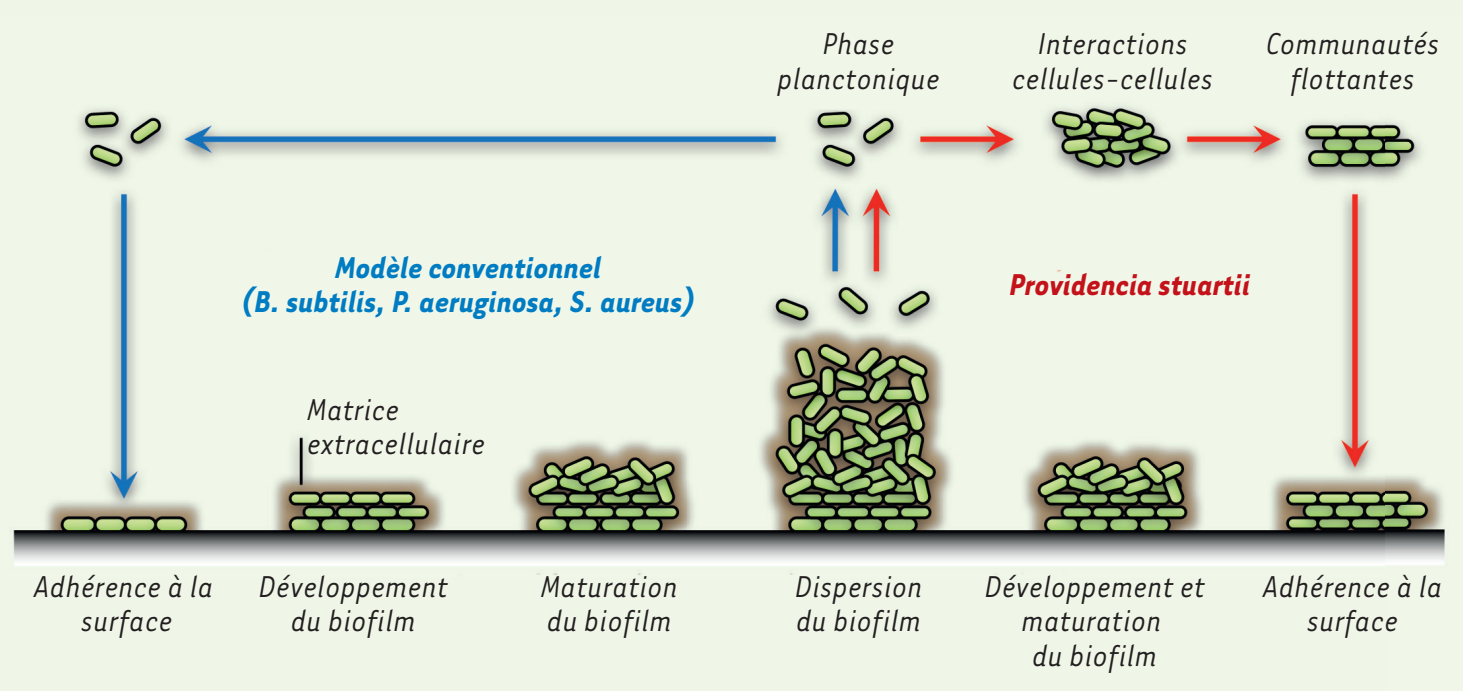

Figure 1. Formation de biofilms. Sur la partie gauche est représenté le modèle conventionnel de formation de biofilms qui prévaut pour $B$. subtilis, $P$. aeruginosa et $S$. aureus. Les bactéries adhèrent à une surface, puis se divisent pour constituer le biofilm. Sur la partie droite est illustré le comportement de Providencia stuartii. Ces bactéries s'assemblent en solution et cette communauté flottante adhère aux surfaces pour ensuite créer un biofilm.

IN urinaires. Les infections causées par P. stuartii sont toujours chroniques et trop souvent mortelles (6 à $33 \%$ de mortalité selon les rapports) du fait de sa forte résistance intrinsèque aux antibiotiques et de sa capacité à former des biofilms. Mais comment ces biofilms s'adaptent-ils aux conditions rencontrées dans le tractus urinaire? Comment la transition phénotypique autorisant le passage de l'état planctonique (libre) au stade sessile (lié à son support) est-elle déclenchée? Quelles sont les protéines impliquées et quelles bases moléculaires exploitent-elles?

Le biofilm de Providencia stuartii, une communauté soudée grâce aux porines Lors de précédents travaux, nous avons démontré que $P$. stuartii n'existe pas, à proprement parler, sous la forme planctonique puisque même en solution, ces bactéries s'assemblent en communautés flottantes [3]. Celles-ci se caractérisent par un contact membranaire étroit entre bactéries adjacentes et représentent ainsi, chez $P$. stuartii, une étape de socialisation nécessaire et préliminaire à celle d'adhérence à la surface en vue de former un biofilm canonique (Figure 1). Cette particularité phénotypique n'est, semble-t-il, pas partagée par d'autres bactéries, telles que Staphylococcus aureus ou Pseudomonas aeruginosa, pour lesquelles la formation de biofilms attachés aux surfaces est le seul mode de socialisation rapporté [4]. Nous avons également démontré que $P$. stuartii était capable de survivre, de croître ainsi que de former et de consolider des biofilms dans des milieux présentant des concentrations élevées en urée, calcium et magnésium, et ceci sur une large gamme de $\mathrm{pH}$, telles que celles rencontrées dans le tractus urinaire. Nous nous sommes ainsi demandé par quels mécanismes $P$. stuartii était capable de détecter ces stress environnants et d'y répondre.

Les porines de diffusion générale ${ }^{2}$ (PDG) constituent plus de $70 \%$ du contenu protéique de la membrane externe bactérienne et représentent la principale porte d'entrée pour les petits solutés hydro-

\footnotetext{
2 Les porines sont des canaux protéiques facilitant le transport des nutriments à travers la membrane externe des bactéries à Gram négatif. Elles sont soit spécialisées, soit de diffusion générale.
}

philes et les ions [5]. Elles semblent donc idéales pour assurer une fonction de senseur. Ces protéines transmembranaires sont généralement associées en trimères fonctionnels au sein desquels chaque monomère présente une structure en tonneau ${ }^{3}$ à 16 brins, interconnectés par de longues boucles extracellulaires (loop L1-L8) et de courts coudes périplasmiques (turn T1-T8) (Figure 2). La plus longue des boucles extracellulaires (L3) se replie dans le canal, créant une zone de constriction qui, par sa charge et sa structure, sert de filtre moléculaire et impose une sélectivité plus ou moins importante aux molécules chargées. Le rôle de cette boucle dans la modification de la perméabilité membranaire aux antibiotiques et la résistance qui y est associée a été démontré $[6,7]$.

$P$. stuartii exprime deux porines au niveau de sa membrane externe, $0 \mathrm{mp}$-Pstl et $0 \mathrm{mp}$-Pst2. Présentant une légère sélectivité pour les anions, $0 \mathrm{mp}$-Pstl est une porine vitale pour la bactérie puisque la

\footnotetext{
3 Les brins $\beta$ ont une torsion intrinsèque et quand huit à trente-six de ces brins $\beta$ sont assemblés, ils peuvent former une structure en tonneau ( $\beta$ barrel).
} 


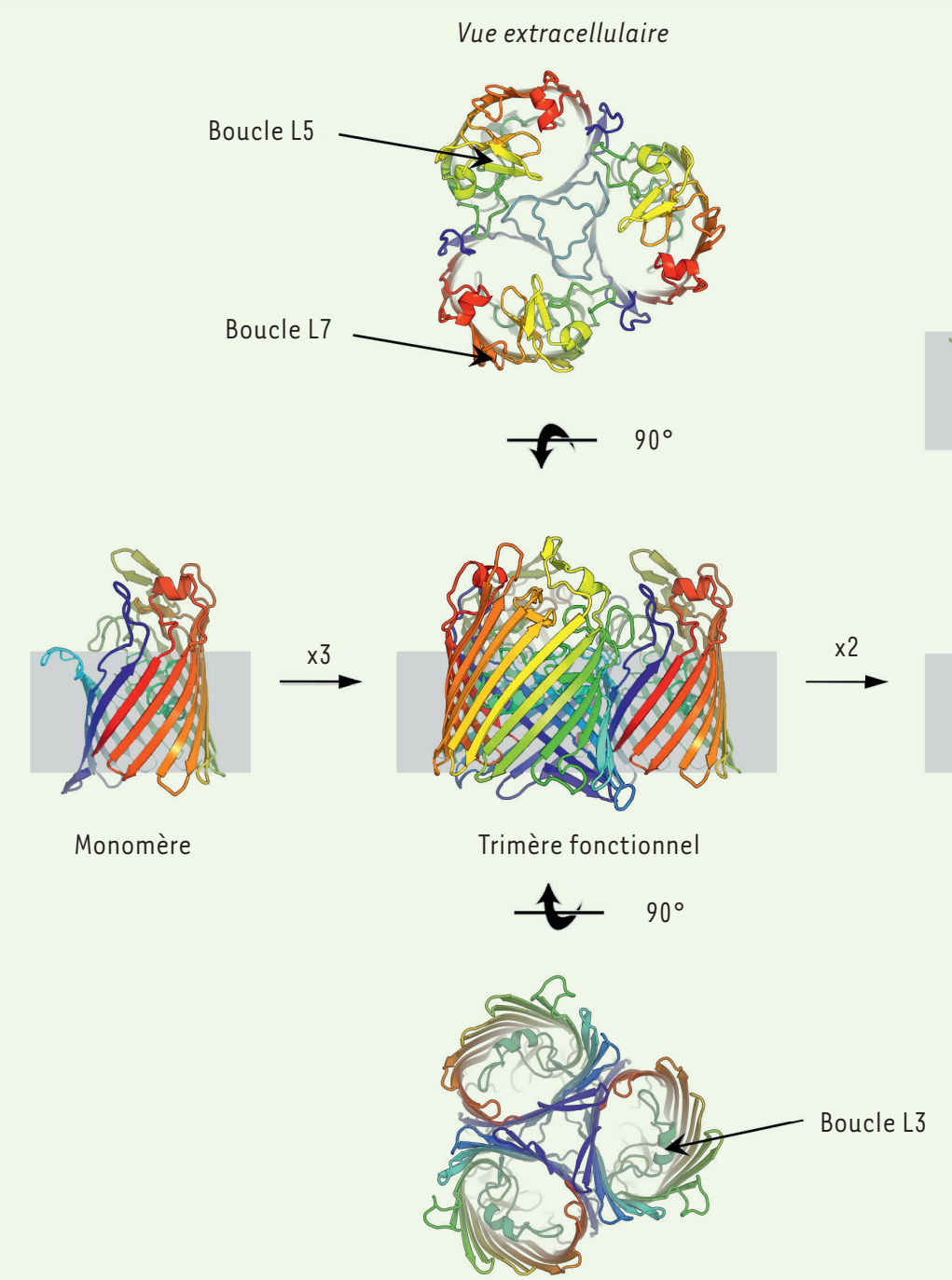

Vue périplasmique

Figure 2. Modèles cristallins des porines. Représentation schématique de la structure cristalline du monomère de porine et de son assemblage en trimère puis en dimère de trimères (DOT) dans la membrane externe (zone grisée) de la bactérie.

délétion du gène codant pour $0 \mathrm{mp}$-Pstl (protéine non produite) est létale. A contrario, 0mp-Pst2 est une porine fortement cation sélective qui, si elle n'est pas vitale, est essentielle durant les phases précoces de croissance et permet une résistance accrue aux stress environnants. Malgré leurs différences, l'expression ectopique ${ }^{4}$ de chacune de ces deux porines dans une souche d'Escherichia

4 Expression à partir d'un plasmide inséré artificiellement dans la bactérie et non à partir du génome. coli dépourvue de ses porines principales (souche BL21 n'exprimant pas les porines $0 \mathrm{mpA}, 0 \mathrm{mpC}, 0 \mathrm{mpF}$ et LamB, appelée BL21 $\Delta 0 \mathrm{mp} 8$ ) permet l'assemblage des bactéries en communautés flottantes, un phénotype comparable à celui observé chez P. stuartii. Cette expression ectopique de l'une ou l'autre des porines ne permet cependant pas l'attachement d' $\varepsilon$. coli à une surface pour former un biofilm canonique, probablement du fait de l'absence d'une matrice auto-sécrétée comme celle produite par $P$. stuartii chez

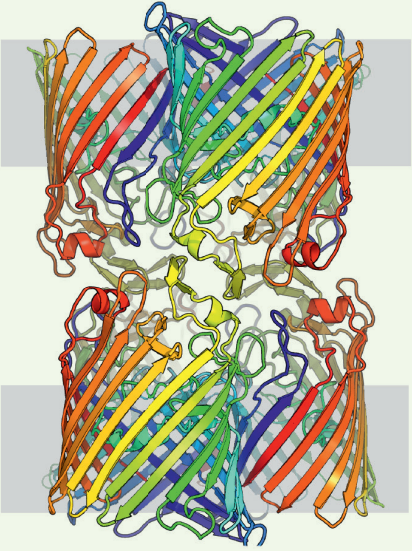

Dimère de trimères (DOT) la souche utilisée. Les porines joueraient ainsi un rôle inédit dans l'induction de la sociabilité bactérienne durant les phases précoces de croissance.

Les porines: une structure complexe pour une fonction inédite

Afin d'établir les bases moléculaires des fonctions diffusives et cohésives des porines de $P$. stuartii, nous avons entrepris de résoudre leur structure à résolution atomique par l'utilisation de la cristallographie aux rayons $X$. Les 


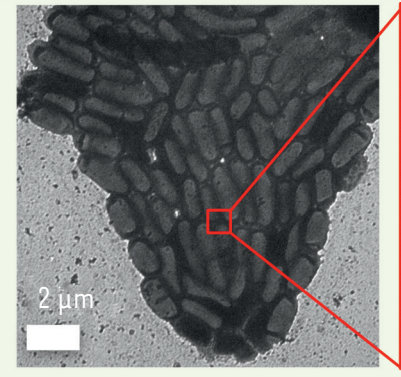

Colonies flottantes

(microscopie électronique à transmission)

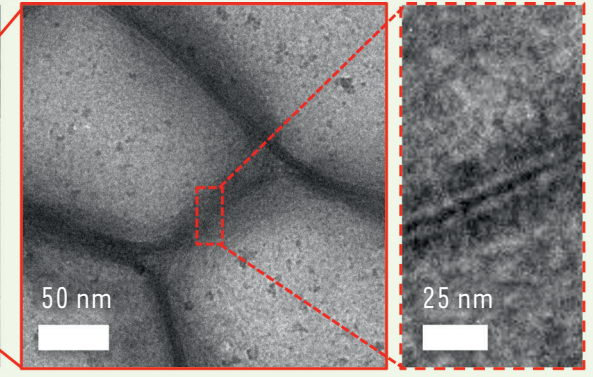

Jonctions membranaires intercellulaires

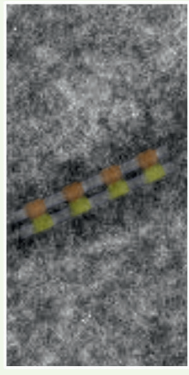

(a)

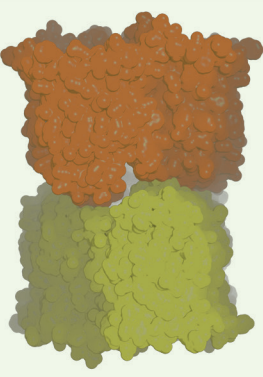

Dimère de trimères de porines (DOT)

Figure 3. Visualisation des dimères de trimères de porine. Des colonies flottantes de Providencia stuarti sont imagées par microscopie électronique à transmission (MET) à différentes résolutions. Le zoom sur les zones de contact intercellulaire montre une distance intermembranaire compatible avec la formation de DOT de porines. Une couleur différente a été attribuée à chaque trimère pour aider à les distinguer sur la superposition d'images. La taille de chaque barre est indiquée sur chacune des images (de $2 \mu \mathrm{m}$ à $25 \mathrm{~nm}$ ). DOT : dimère de trimère.

structures cristallographiques d'OmpPstl et $0 \mathrm{mp}$-Pst2 ont révélé un niveau d'organisation inédit : les trimères de porines s'associent face à face par des interactions auto-complémentaires entre leurs boucles extracellulaires pour former des dimères de trimères (DOT) (Figure 3) [8]. Ces interactions sont de type "steric zipper », un type d'interaction fondé sur la complémentarité de surface et généralement observé au sein des fibres amyloïdes. Pour $0 \mathrm{mp}-$ Pst2, c'est la séquence 282-NLGNYG-287 dans la courte boucle $L 7$ qui contribue à la formation du «steric zipper ». Chez Omp-Pstl, c'est la séquence 206-GVVTSE-211 trouvée dans la longue boucle L5 structurée en épingle de cheveux, qui contribue au «steric zipper ».

Afin de vérifier la capacité de ces porines à s'auto-associer in vitro, nous avons produit des liposomes et avons reconstitué les porines dans la membrane de ces vésicules lipidiques. Nous avons pu observer, grâce à la diffusion dynamique de lumière, une augmentation du rayon hydrodynamique des particules en solution, démontrant que les porines sont capables d'associer les protéoliposomes en agrégats protéoliposomaux. Ces résultats corroborent les données obtenues in vivo et suggèrent un rôle pour ces deux porines dans la formation des contacts intercellulaires et la structuration des communautés flottantes de P. stuartii.

Afin de vérifier que les interactions observées dans les cristaux d'0mpPstl et d'Omp-Pst2 sont à l'origine du contact intermembranaire induit par ces porines, nous avons introduit une ou plusieurs mutations visant à déstabiliser leur «steric zipper » et ainsi perturber leur association en DOT. Nous avons muté la séquence 282-NLGNYG-287 d'OmpPst2 en ajoutant une (G284R/N285G) ${ }^{5}$ ou deux charges positives (G284R/N285K) permettant de supprimer des liaisons hydrogènes stabilisatrices et d'induire des répulsions électrostatiques. Le même raisonnement a été appliqué pour introduire une (D213R) ou deux charges positives (D213R/N293R) dans la séquence d'Omp-Pstl à l'interface du DOT. Comme pour les porines originelles, l'expression ectopique de ces mutants a permis de rétablir la croissance et la viabilité des bactéries $\varepsilon$. coli BL2 $1 \Delta 0 \mathrm{mp} 8$. Cependant, les mutants doublement chargés des deux porines, Omp-Pstl D213R/N293R et

\footnotetext{
${ }^{5}$ La glycine 284 est mutée en arginine et l'asparagine 285 en glycine.

${ }^{6}$ La glycine 284 est mutée en arginine et l'asparagine 285 en lysine.
}

Omp-Pst2 G284R/N285K, se sont révélés incapables de déclencher la formation de larges communautés flottantes, lorsqu'ils ont été exprimés de façon ectopique chez $\varepsilon$. coli. De même, ces mutants ont perdu la capacité d'agréger les protéoliposomes, démontrant que les mêmes interactions sont mises en jeu in vivo, in vitro et in cristallo.

Ainsi, nos données établissent qu'en sus de leurs fonctions diffusives, les porines jouent chez $P$. stuartii un rôle structurel permettant la formation de communautés flottantes grâce à des interactions auto-complémentaires entre leurs boucles extracellulaires. Les canaux des porines restent ouverts dans les structures cristallographiques, suggérant qu'elles conservent leurs fonctions diffusives au sein des DOT. Ces derniers pourraient ainsi jouer un rôle dans la communication intercellulaire au sein des communautés flottantes en sus de leur fonction auto-adhésive. Cette communication passive au travers des DOT de porines pourrait intervenir à chacune des étapes du développement du biofilm de $P$. stuartii, de la formation des communautés flottantes jusqu'à leur sédimentation en micro-colonies et leur adhérence à une surface par sécrétion d'une matrice. 
Conclusions et perspectives:

les porines comme nouvelle cible thérapeutique dans les traitements contre les biofilms?

Nos travaux ont permis d'établir un rôle jusqu'ici insoupçonné des porines dans la socialisation et la formation de biofilms chez $P$. stuartii et de proposer une possible implication dans la communication intercellulaire. Les porines de $P$. stuartii pourraient ainsi servir de jonctions primitives au sein des communautés flottantes formées par la bactérie, sur le modèle des jonctions intercellulaires observées chez les eucaryotes. Les recherches futures viseront à tirer profit des connaissances acquises sur le rôle des porines pour la lutte contre $P$. stuartii, en faisant d'elles des cibles thérapeutiques de choix, permettant à la fois de perturber les échanges transmembranaires de molécules et de disperser les communautés sociales bactériennes dès les étapes précoces de leur développement - et donc avant l'établissement du biofilm canonique. De telles approches peuvent être envisagées en association avec des molécules biocides, telles que les antibiotiques, afin de disperser les cellules des communautés flottantes et des biofilms et ainsi augmenter leur sensibilité aux moyens classiques de lutte antibactérienne [9]. Il faudra également déterminer si d'autres bactéries exploitent les DOT de porines pour se socialiser et échanger ; un rapide examen de la protein data bank ${ }^{7}$ le suggère, révélant que 17 structures de porines génétiquement différentes cristallisent sous la forme de DOT. L'avenir dira combien d'espèces bactériennes exploitent ces structures pour se regrouper et communiquer, permettant de déterminer le spectre d'action d'une stratégie de lutte ciblant les porines. $\diamond$

Porins: a vital role and a social link within the bacterial biofilm of

\section{P. stuartii}

7 La protein data bank (PDB) est la principale banque de dépôt de structures tridimensionnelles (déterminées expérimentalement) de macromolécules biologiques.

\section{LIENS D'INTÉRÊT}

Les auteurs déclarent n'avoir aucun lien d'intérêt concernant les données publiées dans cet article.

\section{RÉFÉRENCES}

1. Hall-Stoodley L, Costerton JW, Stoodley P. Bacterial biofilms : from the natural environment to infectious diseases. Nat Rev Microbiol 2004 ; 2 : 95-108.

2. Lebeaux D, Ghigo JM. Infections associées aux biofilms : quelles perspectives thérapeutiques issues de la recherche fondamentale? Med Sci (Paris) 2012 ; 28 : 727-39.

3. El Khatib M, Tran QT, Nasrallah C, et al. Providencia stuartii form biofilms and floating communities of cells that display high resistance to environmental insults. PLoS One 2017 ; 12 : e0174213.

4. 0'Toole G, Kaplan HB, Kolter R. Biofilm formation as microbial development. Annu Rev Microbiol 2000 ; 54 49-79.

5. Zeth K, Thein M. Porins in prokaryotes and eukaryotes : common themes and variations. Biochem J $2010 ; 431: 13-22$.

6. Eppens EF, Saint N, Van Gelder P, et al. Role of the constriction loop in the gating of outer membrane porin PhoE of Escherichia coli. FEBS Letts 1997 ; 415 : 317-20.

7. Ziervogel BK, Roux B. The binding of antibiotics in OmpF porin. Structure $2013 ; 21: 76-87$.

8. El-Khatib M, Nasrallah C, Lopes J, et al. Porin self-association enables cell-to-cell contact in Providencia stuartii floating communities. Proc Nat Acad Sci USA 2018 ; 115 : ع2220-e8.

9. Fleming D, Rumbaugh $K$. The consequences of biofilm dispersal on the host. Sci Rep 2018; $8: 10738$.

\section{NOUVELLE}

\section{Éléments cis-régulateurs du locus IgH et maturation des lymphocytes B1 et B2}

Hussein Issaoui, Nour Ghazzaui, Mélissa Ferrad, Sandrine Lecardeur, Yves Denizot
UMR CNRS 7276, Inserm U1262, Équipe labellisée Ligue 2018, Université de Limoges, CBRS, Rue Professeur Descottes, 87025 Limoges, France. yves.denizot@unilim.fr
> La fonction d'un lymphocyte B est de produire une immunoglobuline (Ig) spécifique d'un antigène. Pendant bien longtemps rien ne ressemblait plus à un lymphocyte $B$ qu'un autre lymphocyte $B$ alors que le lymphocyte $T$ se déclinait, entre autres, en T4, T8 ou $T \gamma \delta$. En fait, de nombreuses études ont mis en évidence l'hétérogénéité lymphocytaire $B$ avec l'existence des lymphocytes Bl et
B2, compliquée par la description de sous-populations particulières telles les Bla, B1b, B2 folliculaires ou marginaux et $B$ régulateurs. Les lymphocytes Bl participent au système immunitaire inné. Très schématiquement, ils sont produits dans le foie fœtal, vont occuper des niches géographiques précises (entre autres, les muqueuses aériennes et digestives) où ils vont s'y renouveler et produire des IgM et des IgA polyréactives assurant la première ligne de défense contre les pathogènes. Les lymphocytes B2, quant à eux, font partie de l'immunité acquise. Ils sont produits à partir de la moelle osseuse, vont terminer leur maturation dans les organes hématopoïétiques secondaires, produisent des Ig de haute affinité (grâce au processus d'hypermutation soma- 